\title{
Alterations of methionine metabolism in hepatocarcinogenesis: the emergent role of glycine $\mathrm{N}$-methyltransferase in liver injury
}

\author{
Maria M. Similea ${ }^{\text {, Gavinella Latte }}{ }^{\text {, }}$ Claudio F. Feo ${ }^{\text {b }}$, Francesco Feo ${ }^{a}$, Diego F. Calvisia, Rosa M. Pascale ${ }^{a}$ \\ University of Sassari, Sassari, Italy
}

Abstract

The methionine and folate cycles play a fundamental role in cell physiology and their alteration is involved in liver injury and hepatocarcinogenesis. Glycine N-methyltransferase is implicated in methyl group supply, DNA methylation, and nucleotide biosynthesis. It regulates the cellular S-adenosylmethionine/S-adenosylhomocysteine ratio and S-adenosylmethioninedependent methyl transfer reactions. Glycine $\mathrm{N}$-methyltransferase is absent in fast-growing hepatocellular carcinomas and present at a low level in slower growing HCC ones. The mechanism of tumor suppression by glycine $\mathrm{N}$-methyltransferase is not completely known. Glycine N-methyltransferase inhibits hepatocellular carcinoma growth through interaction with Dep domain-containing mechanistic target of rapamycin (mTor)-interacting protein, a binding protein overexpressed in hepatocellular carcinoma. The interaction of the phosphatase and tensin homolog inhibitor, phosphatidylinositol 3,4,5-trisphosphate-dependent rac exchanger, with glycine $\mathrm{N}$-methyltransferase enhances proteasomal degradation of this exchanger by the E3 ubiquitin ligase HectH. Glycine N-methyltransferase also regulates genes related to detoxification and antioxidation pathways. It supports pyrimidine and purine syntheses and minimizes uracil incorporation into DNA as consequence of folate depletion. However, recent evidence indicates that glycine $\mathrm{N}$-methyltransferase targeted into nucleus still exerts strong anti-proliferative effects independent of its catalytic activity, while its restriction to cytoplasm prevents these effects. Our current knowledge suggest that glycine $\mathrm{N}$-methyltransferase plays a fundamental, even if not yet completely known, role in cellular physiology and highlights the need to further investigate this role in normal and cancer cells.

Keywords Hepatocellular carcinoma, Methionine cycle, Folate cycle, tumor suppression, S-adenosylmethionine

Ann Gastroenterol 2018; 31 (5): 1-9

\section{Introduction}

Interest in the role of methionine, its active derivative S-adenosylmethionine (SAM), and the methionine metabolic cycle, in hepatic injury and hepatocarcinogenesis, was stimulated by the discovery of a decrease in SAM levels

Departments of a Clinical, Surgical and Experimental Medicine, Division of Experimental Pathology and Oncology (Maria M. Simile, Gavinella Latte, Francesco Feo, Diego F. Calvisi, Rosa M. Pascale); ${ }^{\mathrm{b} C l i n i c a l}$, Surgical and Experimental Medicine, Division of Surgery (Claudio F. Feo), University of Sassari, Sassari, Italy.

\section{Conflict of interest: None}

Correspondence to: Rosa M. Pascale, Department of Clinical, Surgical and Experimental Medicine, Division of Experimental Pathology and Oncology, University of Sassari, Sassari, Italy; via P. Manzella 4, 07100 Sassari, Italy, e-mail: patsper@uniss.it

Received 12 April 2018; accepted 2 May 2018; published online 12 July 2018

DOI: https://doi.org/10.20524/aog.2018.0288 during liver injury, provoked by ethanol and hepatotoxins, and in hepatocellular carcinoma (HCC), induced by different carcinogens, in animals fed diets containing adequate amounts of methyl donors [1-3]. Reconstitution of the liver's SAM pool by administration of exogenous SAM, prevented liver damage and HCC [3-6]. These pioneering findings were followed by several studies of the mechanisms involved in the antitumor effect of SAM, which directed their attention to the main metabolic cycles implicated in SAM production: the methionine and folate cycles. This led to the discovery of the leading role of glycine N-methyltransferase (GNMT) in liver injury and hepatocarcinogenesis.

\section{The methionine and folate metabolic cycles}

SAM, synthesized from methionine and ATP by methionine adenosyltransferases [7], is the methyl donor for many reactions producing methylated compounds and S-adenosylhomocysteine (SAH), a potent inhibitor of 
most methyltransferases [8] (Fig. 1). SAH is converted to homocysteine and adenosine via a reversible reaction catalyzed by SAH hydroxylase (SAHH). However, the equilibrium of SAHH favors the formation of homocysteine and adenosine. The latter must be transported outside of the cells or metabolized, to avoid homocysteine accumulation. Homocysteine is an amino acid that is not used for protein synthesis, but is an intermediate in methionine metabolism, irreversibly transformed to cystathionine by cystathionine $\beta$-synthetase (CBS), and then to GSH through the transsulfuration pathway, or methylated to form methionine, thus completing the "methionine cycle" (Fig. 1). Homocysteine remethylation may occur during betaine (tri-methylglycine) conversion to di-methylglycine in a reaction catalyzed by betaine-homocysteine methyltransferase (BHMT). Di-methylglycine may be transformed to glycine by a dimethylglycine dehydrogenase (DMGDH) [N,Ndimethylglycine:(acceptor) oxidoreductase (demethylating)] (Fig. 1). Betaine is formed through choline oxidation, catalyzed by choline oxidase, and followed by oxidation of betaine aldehyde by a specific dehydrogenase [9]. In animals, choline may be acquired from the diet and through the transmethylation pathway [10] in which phosphatidylethanolamine is methylated to phosphatidylcholine by phosphatidylethanolamine $\mathrm{N}$-methyltransferase (PEMT) [10] in the presence of SAM (Fig. 1). This pathway is particularly active during choline deficiency [11].

Another source of methionine is the so called "folates cycle" [12] in which 5-tetrahydrofolate (THF) is oxidized to 5,10-methylenetetrahydrofolate (MeTHF), in a reaction catalyzed by sarcosine dehydrogenase (SARDH). MeTHF is transformed to 5-methyltetrahydrofolate (MTHF) by a specific reductase (MeTHFR). Homocysteine plus MTHF leads to methionine and THF in a reaction catalyzed by a 5-methyltetrahydrofolate homocysteine transferase (MHMT) that requires cobalamin as cofactor (Fig. 1).

Glycine methyltransferase (GNMT) plays a fundamental role in methyl group supply, DNA methylation, and nucleotide biosynthesis. GNMT catalyzes the SAM-dependent methylation of glycine to form sarcosine [13] (Fig. 1). SARDH catalyzes glycine resynthesis associated with the formation of MeTHF from THF. GNMT expression regulates the cellular folate status: it binds MTHF and is inhibited by it and increases cellular MTHF retention thus allowing MTHF-dependent remethylation $[14,15]$. GNMT and PEMT are among the largest

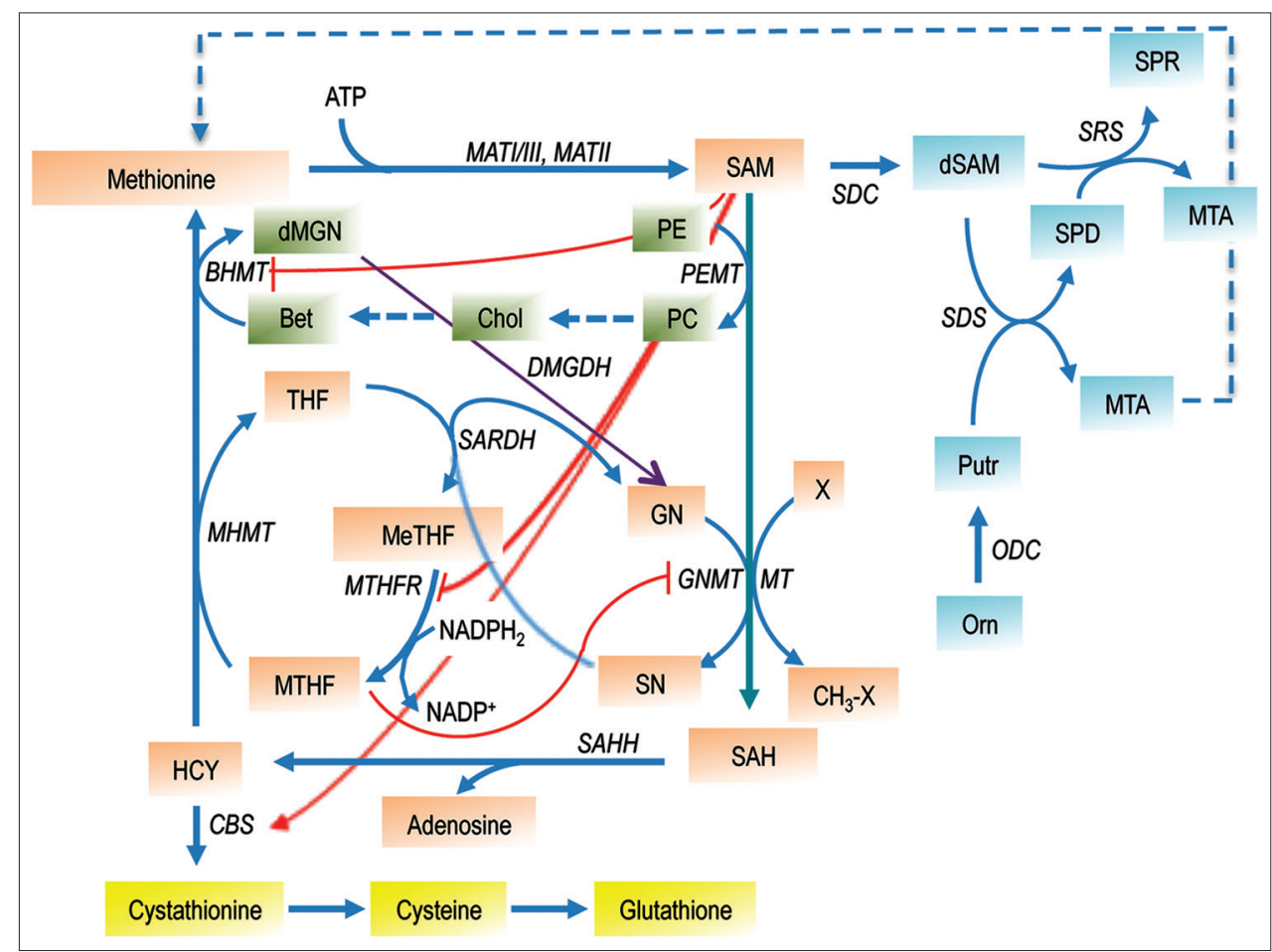

Figure 1 Methionine and folates cycles. Substrates:Bet, betaine;Chol, choline; dMGN, di-methylglycine; dSAM, decarboxylated S-adenosylmethionine; GN, glycine; HCY, homocysteine; MeTHF, 5,10-methylenetetrahydrofolate; MTA, 5-methylthioadenosine; MTHF, 5-methyltetrahydrofolate; Orn, ornithine; PC, phosphatidylcholine; PE, phosphatidylethanolamine; Putr, putrescine; SAH, S-adenosylhomocysteine; SAM, S-adenosylmethionine; $\mathrm{SN}$, sarcosine; SPD, spermidine; SPR, spermine; THF, tetrahydrofolate

Enzymes: BHMT, betaine homocysteine methyltransferase; CBS, cystathionine beta-synthetase; MATI/III, methionine adenosyltransferase I/ III; MATII, methionine adenosyltransferase II; MHMT, methyltetrahydrofolate homocysteine methyltransferase; MT, methyltransferases; MTHFR, 5-methyltetrahydrofolate reductase; ODC, ornithine decarbosylase; PEMT, phosphoethanolamine methyltransferase; SAHH, S-adenosylhomocyteine hydroxylase; SARDH, sarcosine dehydrogenase; SDC, SAM decarboxylase; SDS, spermidine synthetase; SRS, spermine synthetase. The dotted line refers to the "salvage pathway" for methionine biosynthesis. The purple arrow indicates the conversion of dimethyl glycine to sarcosine, the red arrows indicate the inhibition of BHMT and MTHFR and the activation of CBS by SAM, and the inhibition of GNMT by MTHF 
consumers of methyl groups derived from SAM and thus play a major role in homocysteine generation and regulation of methyl group metabolism [16].

Numerous regulatory mechanisms contribute to maintaining an optimal metabolism of folate, methyl groups and homocysteine. The liver is the principal organ in which the excess of methionine is degraded. In it different enzymes, including MATI/III, CBS, BHMT and GNMT, maintain homocysteine at adequate level. Furthermore, the so-called SAM "long-range" interactions [17], which affect enzymes at distance in the metabolic network, induces CBS activation (Fig. 1); this enhances the transsulfuration pathway and inhibits BHMT and MeTHFR (Fig. 1), thus decreasing homocysteine re-methylation to methionine [17]. Indeed, MATI/III Michaelis constant $\left(K_{\mathrm{m}}\right)$ is so high that the elevated methionine liver levels produce large amounts of SAM. High SAM levels inhibit MTHFR while stimulating CBS activity [17,18] (Fig. 1). Therefore, a methionine excess, by producing a SAM excess, induces homocysteine degradation through the transsulfuration pathway, whereas low methionine and SAM levels activate homocysteine re-methylation. Furthermore, SAM allosterically inhibits MeTHFR, thus decreasing the production of 5-methyl-TNF for the subsequent remethylation of homocysteine [19-21] (Fig. 1). SAM also inhibits homocysteine remethylation by inhibiting the expression of BHMT. These SAM effects are associated with the activation of CBS and consequent homocysteine consumption by transsulfuration pathway (Fig. 1).

GNMT binds DMGDH and SARDH (Fig. 1) [21] and is allosterically inhibited by 5-methyl-THF [14,22]. In addition to the allosteric regulation, phosphorylation of GNMT represents an additional post-translational control of this protein [12].

The central role of the methionine and folate cycles in cell metabolism also depends on the multiple interactions of these cycles with cell metabolism. Folate cycle is involved in dTMP synthesis, thus allowing DNA production and synthesis of purine and nucleic acids synthesis [22,23]. Furthermore, SAM decarboxylation by a specific decarboxylase is a fundamental step of polyamine biosynthesis. 5-methylthioadenosine (MTA), an end-product of this biosynthesis, inhibits SAMdecarboxylase [2]. This control step is regulated by the activity of MTA phosphorylase, which catalyzes the transformation of MTA into 5-methylthioribose and adenine, allowing the methionine resynthesis in the "salvage pathway" (Fig. 1).

The SAM/SAH ratio plays a key role in SAM-dependent methyltransferase reactions. MATI and MATIII isozymes, respectively the tetramer and dimer of the subunit a 1 isozymes, are encoded by the liver-specific MAT1A gene [24] (Fig. 1). The widely distributed MATII isoform (the $\alpha 2$-subunit) is codified by the MAT2A gene, whose expression, prevalent in fetal liver, is gradually replaced by MAT1A in adult liver [24]. The $K_{\mathrm{m}}$ for methionine of MATI and MATIII are $23 \mu \mathrm{M}-1 \mathrm{mM}$ and $215 \mu \mathrm{M}-7 \mathrm{mM}$, respectively. Therefore, SAM, at physiological liver level (0.1-0.2 $\mu \mathrm{mol} / \mathrm{g}$ of liver, about $60 \mu \mathrm{M})$ slowly inhibits MATI and stimulates MATIII activity. In contrast, MATII, with a lower $\mathrm{K}_{\mathrm{m}}$ for methionine $(4-10 \mu \mathrm{M})$, is inhibited by its reaction product [24]. A third gene, MAT2B, encodes the MATs $\beta$-subunit, devoid of catalytic activity, which regulates MATII by lowering its $\mathrm{K}_{\mathrm{m}}$ for methionine and inhibition constant $\left(K_{\mathrm{i}}\right)$ for SAM [25]. Therefore, the $\beta$-subunit association with the $\alpha$-subunit renders MATII more susceptible to inhibition by SAM [25].

GNMT is a major regulator of the cellular SAM/SAH ratio and SAM-dependent methyl transfer reactions. Low SAM levels favor homocysteine remethylation, whereas high SAM levels activate CBS. Furthermore, GNMT provides an alternative route for the conversion of SAM excess to SAH and the preservation of the SAM/SAH ratio. GNMT is abundant in liver and its product sarcosine, which has no known physiological role, is converted back to glycine by sarcosine dehydrogenase [14,21,22]. GNMT is a major regulator of the cellular SAM/SAH ratio and SAM-dependent methyl transfer reactions. SAM-dependent methyltransferases are generally strongly inhibited by the product SAH, and the cellular SAM/ SAH ratio plays a key role in methyl-transfer reactions [14]. Unlike most SAM-dependent methyltransferases, GNMT has a relatively high $\mathrm{K}_{\mathrm{m}}$ value for SAM and is weakly inhibited by SAH: the $K_{\mathrm{i}}$ values of GNMTs for SAH are $35-80 \mu \mathrm{M}$, much higher than those for other methyltransferases [14,21]. Therefore, at physiological levels of SAM (0.1-0.2 $\mu \mathrm{mol} / \mathrm{g}$ of liver) and SAH (0.02-0.06 $\mu \mathrm{mol} /$ of liver), GNMT exhibits appreciable activity. Therefore, the fluctuations in GNMT activity could alter the SAM/SAH ratio, thus influencing the activity of methyltransferases.

Further, GNMT, as a major hepatic folate binding protein, binds to and may be inhibited by MTHF [14-17,22]. Therefore, when SAM levels increase, MeTHFR inhibition leads to a decrease in free MTHF and dissociation of the complex GNMT-MTHF $[14,22]$. The consequent rise in free GNMT prevents the SAM level from rising excessively. Conversely, when SAM concentration tends to decrease, the amount of free GNMT falls, MeTHFR inhibition is released and more MTHF is available. Thus, GNMT increases the cells' folate content and the remethylation of MTHFR-dependent homocysteine. For this reason, the GNMT pathway may be considered a "salvage pathway".

\section{Alterations of the methionine cycle in liver cancer}

Decreases in SAM liver content characterize liver injuries that lead to inflammation, necrosis, cirrhosis and eventually cancer, such as alcoholic steatohepatitis [26,27], and D-galactosamine [28], acetaminophen [29] and $\mathrm{CCl}_{4}[30,31]$ toxicity. In cirrhotic liver, the oxidation of cysteine residue at the ATP binding site is involved in MATI/III downregulation, decrease in MAT1A: MAT2A ratio (MAT1A/MAT2A switch), and SAM and GSH contents [32,33]. This favors hepatic oxidative stress and fibrosis followed by the development of HCC. These alterations could be partly attributable to the absence of prohibitin (PHB)-1. This, together with PHB-2, forms multimeric ring complexes in the mitochondrial inner membrane and plays a role in mitochondrial maturation [34,35]. SAM contributes to PHB1 stability and a drop in SAM is 
associated with PHB1 deficiency [36]. This deficiency leads to increases in the production of reactive oxygen species, impairs the formation of mitochondrial respiratory supercomplexes, disorganizes the mitochondrial cristae morphology, and increases the sensitivity to stimuli-elicited apoptosis [37].

The strong involvement of the MAT1A/MAT2A switch and the fall in SAM levels during hepatocarcinogenesis is further demonstrated by the observation that the Mat1A-KO mouse model, which features a chronic SAM deficiency that is not compensated for by MAT2A induction, is characterized by hepatomegaly at 3 months of age, macrovesicular steatosis involving 25-50\% of hepatocytes, mononuclear cell infiltration in periportal areas, at 8 months, and HCC development at 18 months [38].

A sharp decrease in SAM content and SAM/SAH ratio has been documented in liver of rats fed an adequate diet, during hepatocarcinogenesis induced by different carcinogens, and is still present in dysplastic nodules (DN) and HCC several weeks after carcinogen administration is discontinued [3-5]. Decreases in SAM content and SAM/SAH ratio also occur in human HCC and, to a lower extent, in the cirrhotic liver surrounding a tumor [39]. SAM administration reconstitutes the GSH pool, has beneficial effects against liver fibrosis in both rats and humans $[24,31]$ and prevents HCC development in rats and mice [3-6,39].

The genesis of the MAT1A/MAT2A switch is complex. A mechanism deals with changes of promoter methylation of the MAT1A and MAT2A genes in HCC [40-43]. Methylation of CCGG sequences of MAT1A promoter, associated with a decrease in MAT1A, occurs in cirrhotic liver of $\mathrm{CCl}_{4}$-treated rats and in HepG2 and Huh7 liver cancer cell lines [40]. MAT1A expression is reactivated in the human hepatoblastoma cell line HepG2 treated with the demethylating agent 5-aza-2'deoxycytidine or the histone deacetylase inhibitor trichostatin, suggesting a role for DNA hypermethylation and histone deacetylation in MAT1A silencing [40]. In HuH7 cells, MAT1A downregulation is also associated with CCGG methylation at +10 and +80 of the coding region [41]. Conversely, MAT2A upregulation in human HCC is associated with CCGG hypomethylation of the gene promoter $[42,43]$.

The highest MAT1A promoter hypermethylation and MAT2A promoter hypomethylation were found in human HCC with poorer prognosis (HCCP), based on survival time after partial liver resection, whereas, in human HCC with a better prognosis (HCCB) and small changes in the MAT1A: MAT2A ratio, $\mathrm{CpG}$ methylation and histone $\mathrm{H} 4$ acetylation occurred [43]. Interestingly, the Mat1A/Mat2A switch and low SAM levels associated with CpG hypermethylation and histone $\mathrm{H} 4$ deacetylation in Mat1A promoter, and prevalent CpG hypomethylation and histone $\mathrm{H} 4$ acetylation in Mat2A promoter has also been found in fast growing HCC of Fisher 344 (F344) rats, whereas these alterations were almost absent in slowly growing HCC of brown Norway (BN) rats [43].

Post-transcriptional mechanisms are also involved in MAT1A: MAT2A switch. Among the mRNA-binding proteins (RBPs), AUF1, increases mRNA decay, whereas HuR binds to AUrich elements promoting mRNA stabilization [43-45].
A Mat1A mRNA decrease in fetal rat liver is associated with increased interactions with AUF1, whereas a Mat2A mRNA increase is associated with its interaction with $\mathrm{HuR}$ $[43,46]$. A sharp increase in AUF1 and HuR occurs in F344 and human HCC associated with a consistent increase in MAT1A-AUF1 and MAT2A-HuR ribonucleoproteins in both HCC types [43].

The importance of these findings also lies in the fact that rats F344 and $\mathrm{BN}$ are, respectively, genetically susceptible and resistant to the development of liver tumors. Numerous loci responsible for the resistance to the development of dysplastic nodules and carcinomas whose effect predominates in resistant rats have been identified [reviewed in 47]. These observations indicate that the alterations of promoter methylation of Mat1A and Mat2A genes and histone deacetylation, responsible for the Mat1A: Mat2A switch, are genetically determined.

Recent data indicate that miRNAs may also contribute contribute to the MAT1A: MAT2A switch. Knockdown of miR-664, miR-485-3p, and miR-495 individually in Hep3B and HepG2 cells was found to induce MAT1A expression, and Hep3B cells tumorigenesis in nude mice was decreased by stable overexpression of these miRNAs and increased by their knockdown [48].

\section{GNMT in liver injury}

GNMT was first described by Blumenstein and Williams in guinea pig liver [13]. GNMT gene expression is greatest in mammalian liver, followed by the acinar tissue of pancreas and kidney [49]. The presence of GNMT protein and enzymatic activity has been demonstrated in liver [49]. This protein is also expressed in fetal and adult rabbit liver and in rat liver, pancreas and prostate $[50,51]$.

GNMT mRNA is downregulated in cirrhotic liver and in HCC induced by hepatitis $\mathrm{C}$ virus infection and alcohol abuse [52,53]. Recent findings showed that GNMT deficiency in mice induces steatohepatitis, fibrosis, cirrhosis, and HCC. A lack of GNMT triggers natural killer cell activation in GnmtKO mice and depletion of TRAIL-producing natural killer cells significantly attenuates acute liver injury and inflammation in these animals [54]. In contrast, 1,2,3,4,6-penta-O-galloyl- $\beta$ d-glucopyranoside, a GNMT inducer, has potent anti-HCC effects both in vitro and in vivo [55]. Interestingly, GNMT is completely absent in fast-growing HCC and present at a low level in slower growing HCC, which suggests that it has an impact on tumor progression [50].

Epigenetic silencing of the GNMT gene, due to promoter methylation was found in pancreatic adenocarcinoma [56]. Hypermethylation of GNMT gene also occurs in some HCC cell lines and in $20 \%$ of primary HCCs [57]. Furthermore, GNMT binds different carcinogens including polyaromatic hydrocarbons and aflatoxins and prevents the DNA adduct formation and cytotoxicity induced by these carcinogens $[58,59]$. Studies conducted in Taiwanese men, suggested that GNMT is a tumor susceptibility gene for 
HCC [60] and prostate cancer [61]. However, in another study [62], GNMT short tandem repeat polymorphism (STRP1), and single nucleotide polymorphism rs10948059 were not associated with an increase in the risk of prostate cancer in Taiwanese men of European descent. An androgen response element within the first exon of the GNMT gene, which binds androgen receptor in vitro and in vivo, was identified in prostate cancer, which highlights androgen as an important feature of GNMT regulation $[63,64]$.

\section{Role of GNMT in hepatocarcinogenesis}

A study of Gnmt-KO mice by Luka et al [65] made a crucial contribution to our recognition of the role of GNMT in hepatocarcinogenesis. In that study, the absence of liver Gnmt resulted in increases of up to a 7 -fold and 35-fold in free methionine and SAM, respectively, while SAH level decreased 3-fold. Further studies using a GnmtKO mouse model with knockout of the 1-5 exons in Gnmt gene also resulted in HCC development [66,67]. These mice showed hypermethioninemia and elevated levels of serum aminotransferase and hepatic SAM. About $60 \%$ of these mice exhibited increased glycogen storage in the liver. HCC developed between 14-24 months of age, with a prevalence of female mice [67]. Wnt signaling pathway, global DNA hypomethylation and aberrant expression of DNA methyltransferases 1 and $3 b$ were found even at early stages of HCC development [67]. Gadd45a, Pak1, Mapk3 and Dusp3 genes of the MAPK pathway were activated in GnmtKO mice, especially females [67].

Elevated serum aminotransferase, methionine, and SAM levels, associated with the development of liver steatosis and fibrosis were found by Martínez-Chantar et al [68] in a male Gnmt-KO model in which GNMT exon 1 was disrupted. HCCs developed at the age of 8 months [69]. Activation of Ras and Jak/Stat pathways and suppression of the Ras inhibitors, Rasassociation domain family/tumor suppressor (RASSF) 1 and 4, and of the Jak/Stat inhibitors, SOCS 1-3 and cytokine-inducible $\mathrm{SH} 2$-protein, occurred in these mice [68]. Hypermethylation of Rassf1 and Socs 2 promoters and binding of trimethylated lysine 27 of histone 3 to these two genes occurred in HCC from GnmtKO mice [68] indicating that GNMT loss induced aberrant methylation of DNA and histones resulting in epigenetic modulation of critical carcinogenic pathways. The reasons for the discrepancies between the two Gnmt-KO models are not clear and require additional investigation.

Some interesting relationships also occur between GNMT and LKB1/AMPK signaling [69]. Hepatoma cells from GnmtKO mice lacking exon 1 exhibit, like human HCC, increased activity of $L k b 1$ and Ras. Ras-mediated Lkb1 hyper-activation promotes Ampk activation and proliferation of GNMT-KO hepatoma cells, and requires Erk2 and p90rsk activity and Rasgrp3 expression. Noticeably, reduced levels of GNMT, phosphorylation of AMPKa at Thr172, and increased levels of RAS, LKB1 and RASGRP3 were found, in HCC samples from patients with short survival.

\section{Mechanisms of the inhibition of hepatocarcinogenesis by GNMT}

GNMT impacts on HCC growth through different mechanisms (Fig. 2). It inhibits HCC growth through interaction with Dep domain-containing mtor-interacting protein (DEPTOR), an mTOR binding protein overexpressed in HCC, especially in patients with poor prognosis [70]. GNMT overexpression causes a decrease in Mechanistic target of rapamycin (mTORC1) signaling, delays G2/M cell cycle progression, and inhibits $\mathrm{HuH}-7$ cell proliferation [70]. DEPTOR over-expression activates AKT via the inhibition of the negative feedback loop from S6K to PI3K [71]. Knockdown of DEPTOR expression in HuH-7 cells causes activation of the mTORC1 targets S6K and 4E-BP, but suppresses AKT and reduces cell survival [70].

Recent evidence [72] revealed a new mechanism of the interaction between GNMT and AKT signaling. It was observed that the interaction of the phosphatase and tensin homolog (PTEN) inhibitor phosphatidylinositol 3,4,5-trisphosphatedependent Rac exchanger 2 (PREX2) with GNMT enhances PREX2 proteasomal degradation by the E3 ubiquitin ligase HectH9 (Fig. 2). GNMT or HectH9 depletion resulted in AKT activation in a PREX2 dependent manner and enhanced cell proliferation. Elevated Prex2 protein expression and

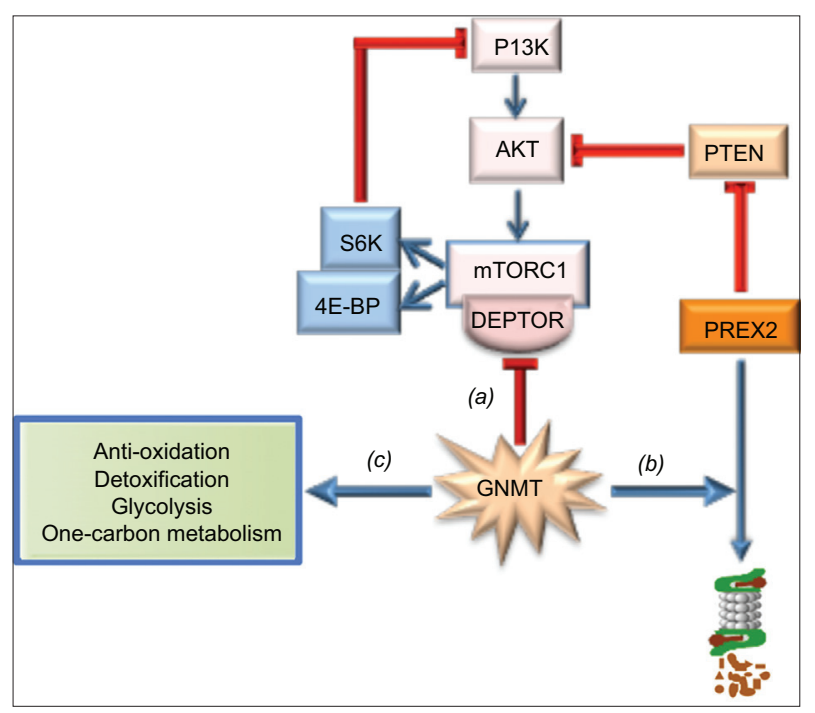

Figure 2 Mechanisms of inhibition of HCC growth by GNMT. (a) The inhibition of DEPTOR, with consequent activation of mTORC1 targets SK6 and 4E-BP, allows the feedback negative effect of SK6 on the PI3K/ AKT axis. (b) GNMT impedes the proteasomal degradation of PREX2 by the $\mathrm{E} 3$ ubiquitin ligase $\mathrm{HecH}$, thus allowing the inhibition, by PREX2, of the AKT inhibitor PTEN. (c) The maintenance of adequate levels of proteins involved in the anti-oxidation/detoxification response, glycolytic energy metabolism and one-carbon metabolism pathways may impede oxidative stress and liver damage that should favor HCC development

$A K T$, murine thymoma viral oncogene homolog; DEPTOR, dep domain-containing mtor-interacting protein; GNMT, glycine $\mathrm{N}$-methyltransfertase; mTORC1, mechanistic target of rapamycin complex 1; PI3K, phosphatidylinositol 3-kinase; PTEN, phosphatase and tensin homolog 
AKT activation occur in GNMT-KO mice liver. Interestingly, upregulation of PREX2 protein expression in human HCC is associated with poorer survival. No changes in PREX2 mRNA level were found, suggesting a post-translational alteration of PREX2 expression [72].

GNMT was also found to maintain normal levels of proteins related to detoxification and antioxidation pathways (Fig. 2). Proteins involved in the anti-oxidation/detoxification response, glycolytic energy metabolism and one-carbon metabolism pathways were found to be downregulated significantly and peroxidation was increased in Gnmt-KO mice with respect to wild mice [73]. In contrast, the survival of $\mathrm{HuH}-7$ cells overexpressing GNMT, treated with $\mathrm{H}_{2} \mathrm{O}_{2}$, has been found to be significantly higher than that of controls [73].

The strong involvement of GNMT in cellular folate metabolism [14,22,23] may partly account for the clinical behavior of GNMT deficiency. Hepatic folate increases in Gnmt-TG mice whereas it decreases in Gnmt-KO mice [22]. GNMT overexpression, in addition to the increase in folate concentration, induces folate-dependent homocysteine methylation [23], which contrasts with a reduction in MHMT (Fig. 3) expression in Gnmt-KO mice, indicating that the GNMT deletion impairs methylfolate-dependent metabolism [22]. MeTHFR catalyzes the conversion of 5,10-methyltetrahydrofolate to 5-methyltetrahydrofolate (Fig. 3). The latter inhibits GNMT activity (Fig. 1) [74]. SAM accumulation in Gnmt-KO mice results in Methfr down-regulation with a consequent decrease in the supply of methyl groups from folate metabolism $[18,19]$. Also, formiminotransferase cyclodeaminase (FTCD; Fig. 3) - which links histidine catabolism to folate metabolism and catalyzes the folate-dependent degradation of $\mathrm{N}$-formiminoglutamic acid to form 5,10-methylenetetrahydrofolate, glutamate, and ammonia - is downregulated in the absence of GNMT activity [75]. These findings are in keeping with the presence in Gnmt-KO mice of a phenotype similar to that of patients with congenital GNMT deficiencies $[75,76]$.

Studies of GNMT in human HCC have shown that GNMT gene alteration, including insertion/deletion polymorphism in the promoter region, is an early event in HCC development and that GNMT could be considered a new tumor susceptibility gene for HCC $[61,77]$. In respect to these findings, it is interesting the observation that some genes involved in methionine metabolism, such as BHMT and GNMT, are connected to the genetic susceptibility to liver cancer [77]. Chemically induced hepatocarcinogenesis in genetically susceptible F344 rats is characterized by the development of numerous $\mathrm{DN}$ that progress to HCCs, whereas the same treatments induce in the genetically resistant $\mathrm{BN}$ rats only a few slow-proliferating DN and HCCs [77]. A gene expression signature characterized by highest expression of the oncosuppressors Csmd1, Dmbt1, Dusp1, and Gnmt, in DN, and Bhmt, Dmbt1, Dusp1, Gadd45g, Gnmt, Napsa, Pp2ca, and Ptpn13, in HCCs, was identified in resistant rats. Greater aggressiveness linked to higher cell cycle

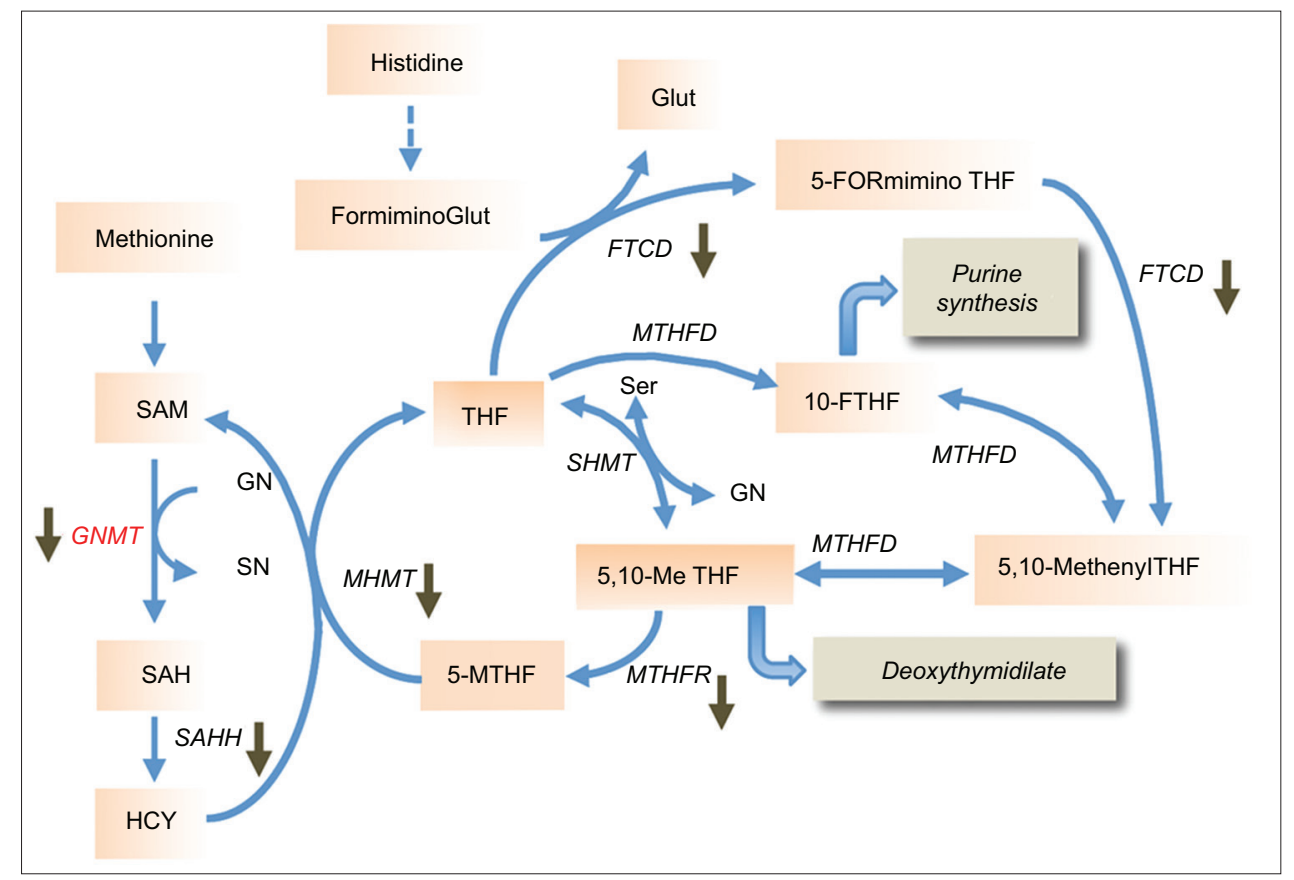

Figure 3 Effect of gnmt deficiency on the folate metabolism

Substrates: formiminoglut, formiminoglutamate; 5-formimino-thf, 5-formimino-tetrahydrofolate; 10-FTHF, 10-formyltetrahydrofolate; Glut, glutamate; GN, glycine; HCY, homocysteine; 5,10-methenylTHF, 5,10-methenyletrahydrofolate; 5,10-MeTHF, 5,10-methylenetetrahydrofolate; 5-MTHF, 5-methyltetrahydrofolate; SAH, S-adenosylhomocysteine; SAM, S-adenosylmethionine; SN, sarcosine; Ser, serine; THF, tetrahydrofolate Enzymes: FTCD, formiminotransferase cyclodeaminase; GNMT, glycine methyltransferase; MHMT, methyltetrahydrofolate homocysteine methyltransferase; MTHFD, methylenetetrahydrofolate dehydrogenase; MTHFR, 5-methyltetrahydrofolate reductase SAHH, S-adenosylhomocyteine hydroxylase; SHMT, serine hydroxymethyl transferase. The black arrows indicate the decrease in enzyme activities linked to that of GNMT activity 
and signal transduction pathways activation was documented in human HCCP with respect to HCCB [77-79]. Integrated gene expression data revealed the highest expression of proliferation-related $C T G F, c-M Y C$, and PCNA, and the lowest expression of BHMT, DMBT1, DUSP1, GADD45g, and GNMT, in more aggressive rat and human HCC [77]. These findings confirm that genes involved in the methionine cycle, such as $B H M T$ and GNMT, contribute to determining the prognosis of HCC.

The mechanism by which GNMT can participate in tumor prevention/suppression in humans is not completely clear. It has been shown [80] that GMMT supports methylene-folate dependent pyrimidine synthesis and formyl-folate dependent purine syntheses (Fig. 3). It also minimizes uracil incorporation into DNA as a consequence of folate depletion and translocates into nuclei during prolonged folate depletion. According to these findings, loss of GNMT impairs nucleotide biosynthesis, whereas GNMT overexpression enhances nucleotide biosynthesis and improves DNA integrity by reducing uracil misincorporation in DNA both in vitro and in vivo [80].

Intriguingly, recent observations [81] indicate that GNMT has a secondary function, as a regulator of cellular proliferation, independent of its catalytic activity. According to this study, GNMT is strongly downregulated in human cancers and is undetectable in cancer cell lines while the transient expression of the protein in cancer cells induces apoptosis and the activation of ERK1/2 as an early pro-survival response. The anti-proliferative effect of GNMT is partially reversed by the pancaspase inhibitor zVAD-fmk. High GNMT levels were found in regenerating liver and in NIH3T3 mouse fibroblasts, where they did not produce cytotoxic effects. Notably, GNMT, a predominantly cytoplasmic protein, translocates into nuclei upon transfection of cancer cells [81]. The induction of apoptosis was associated with the GNMT nuclear localization but was independent of its catalytic activity or folate binding. GNMT targeted to nuclei still exerted strong anti-proliferative effects while its restriction to cytoplasm prevented these effects. Thus, according to these findings, the oncosuppressive action of GNMT would be independent of its catalytic activity.

\section{Concluding remarks}

Increasing evidence indicates that GNMT plays an essential regulatory role in liver cells. Its regulation of methionine and folate cycles is fundamental. GNMT preserves DNA integrity by promoting MeTHF-dependent pyrimidine synthesis and 10-formylTHF-dependent purine synthesis, it thus contributes to reducing uracil misincorporation in DNA and regulates genes related to detoxification and anti-oxidation pathways. Furthermore, GNMT may reduce the proliferative activity of HCC by controlling the Pi3K/AKT pathway through interaction with DEPTOR, an activator of AKT signaling, and enhancing the degradation of the PTEN inhibitor PREX2. In addition, WNT and MAPK signaling and Cyclin D1 and c-Myc genes are activated in GNMT-KO mice. However, the mechanisms through which GNMT exerts an inhibitory effect on these and others signaling pathways contributing to HCC growth are poorly known and need further investigation. Further studies should be devoted to the mechanisms of the oncosuppressor effect of the nuclear GNMT localization. Finally, the discovery of insertion/deletion polymorphisms in the promoter region of GNMT and the association of low GNMT expression with genetic susceptibility to HCC suggest that GNMT is implicated in the genetic susceptibility to this tumor. This is an attractive possibility that needs further experimental support. Overall, current information suggests that GNMT plays a basic, even if not yet completely known, role in the cellular physiology and highlights the need to investigate this role further in normal and cancer cells.

\section{Acknowledgment}

Supported by funds from Fondazione Sardegna.

\section{References}

1. Feo F, Garcea R, Pascale RM, Pirisi L, Daino L, Donaera A. The variations of S-adenosyl-L-methionine content modulate hepatocyte growth during phenobarbital promotion of diethylnitrosamine-induced rat liver carcinogenesis. Toxicol Pathol 1987; 15:109-114.

2. Garcea R, Pascale RM, Daino L, et al. Variations of ornithine decarboxylase activity and S-adenosyl-L-methionine and 5'-methylthioadenosine contents during the development of diethylnitrosamine-induced liver hyperplastic nodules and hepatocellular carcinomas. Carcinogenesis 1987;8:653-658.

3. Pascale RM, Simile MM, Satta G, et al. Comparative effects of L-methionine, S-adenosyl-L-methionine and 5 -methylthioadenosine on the growth of preneoplastic lesions and DNA methylation in rat liver during the early stages of hepatocarcinogenesis. Anticancer Res 1991;11:1617-1624.

4. Garcea R, Daino L, Pascale RM, et al. Inhibition of promotion and persistent nodule growth by S-adenosyl-L-methionine in rat liver carcinogenesis: role of remodeling and apoptosis. Cancer Res 1989;49:1850-1856.

5. Pascale RM, Simile MM, De Miglio MR, et al. Chemoprevention by S-adenosyl-L-methionine of rat liver carcinogenesis initiated by 1,2-dimethylhydrazine and promoted by orotic acid. Carcinogenesis 1995; 16:427-430.

6. Lu SC, Ramani K, Ou X, et al. S-adenosylmethionine in the chemoprevention and treatment of hepatocellular carcinoma in a rat model. Hepatology 2009;50:462-471.

7. Cantoni GL. Activation of methionine for transmethylation. J Biol Chem 1951;189:745-754.

8. Cantoni GL. Methylation of nicotinamide with soluble enzyme system from rat liver. J Biol Chem 1951;189:203-216.

9. Li Z, Vance DE. Phosphatidylcholine and choline homeostasis. J Lipid Res 2008;49:1187-1194.

10. Bremer J, Greenberg DM. Biosynthesis of choline in vitro. Biochim Biophys Acta 1960;37:173-175.

11. Pascale R, Pirisi L, Daino L, et al. Role of phosphatidylethanolamine methylation in the synthesis of phosphatidylcholine by hepatocytes isolated from choline-deficient rats. FEBS Lett 1982;145:293-297.

12. Blom HJ, Smulders Y. Overview of homocysteine and folate 
metabolism. With special references to cardiovascular disease and neural tube defects. J Inherit Metab Dis 2011;34:75-81.

13. Blumenstein J, Williams GR. Glycine methyltransferase. Can J Biochem Physiol 1963;41:201-210.

14. Cook RJ, Wagner C. Glycine N-methyltransferase is a folate binding protein of rat liver cytosol. Proc Natl Acad Sci U S A 1984;81:3631-3634.

15. Wang YC, Wu MT, Lin YJ, Tang FY, Ko HA, Chiang EP. Regulation of Folate-Mediated One-Carbon metabolism by Glycine N-Methyltransferase (GNMT) and Methylenetetrahydrofolate reductase (MTHFR). J Nutr Sci Vitaminol (Tokyo) 2015;61(Suppl):S148-150.

16. Williams KT, Schalinske KL. New insights into the regulation of methyl group and homocysteine metabolism. I Nutr 2007; 137:311-314.

17. Reed MC, Gamble MV, Hall MN, Nijhout HF. Mathematical analysis of the regulation of competing methyltransferases. $B M C$ Syst Biol 2015;9:69.

18. Finkelstein JD, Martin JJ. Inactivation of betaine-homocysteine methyltransferase by adenosylmethionine and adenosylethionine. Biochem Biophys Res Commun 1984;118:14-19.

19. Kutzbach C, Stokstad ELR. Feedback inhibition in methylenetetrahydrofolate reductase in rat liver by S-adenosylmethionine. Biochim Biophys Acta 1967;139:217-220.

20. Jencks DA, Matthews RG. Allosteric inhibition of methylenetetrahydrofolate reductase by adenosylmethionine. Effects of adenosylmethionine and NADPH on the equilibrium between active and inactive forms of the enzyme and on the kinetics of approach to equilibrium J Biol Chem 1987;262:2485-493.

21. Ou X, Yang H, Ramani K, Ara AI, Chen H, Mato JM, Lu SC. Inhibition of human betaine-homocysteine methyltransferase expression by S-adenosylmethionine and methylthioadenosine. Biochem J 2007;401:87-96.

22. Wagner C, Briggs WT, Cook RJ. Inhibition of glycine $\mathrm{N}$-methyltransferase activity by folate derivatives: implications for regulation of methyl group metabolism. Biochem Biophys Res Commun 1985;127:746-752.

23. James SJ, Miller BJ, Cross DR, McGarrity LJ, Morris SM. The essentiality of folate for the maintenance of deoxynucleotide precursor pools, DNA synthesis, and cell cycle progression in PHA-stimulated lymphocytes. Environ Health Perspect 1993;101(Suppl 5):173-178.

24. Ramani K, Mato JM, Lu SC. Role of methionine adenosyltransferase genes in hepatocarcinogenesis. Cancers (Basel) 2011;3:1480-1497.

25. LeGros L, Halim AB, Chamberlin ME, Geller A, Kotb M. Regulation of the human MAT2B gene encoding the regulatory beta subunit of methionine adenosyltransferase, MAT II. J Biol Chem 2001;276:24918-24924.

26. Feo F, Pascale R, Garcea R, et al. Effect of the variations of $\mathrm{S}$-adenosyl-L-methionine liver content on fat accumulation and ethanol metabolism in ethanol-intoxicated rats. Toxicol Appl Pharmacol 1986;83:331-341.

27. Lieber CS, Casini A, DeCarli LM, et al. S-adenosyl-L-ethionine attenuates alcohol-induced liver injury in the baboon. Hepatology 1990;11:165-172.

28. Stramentinoli G, Gualano M, Ideo G. Protective role of S-adenosylmethionine on liver injury induced by $\mathrm{D}$-galactosamine in rats. Biochem Pharmacol 1978;27:1431-1433.

29. Stramentinoli G, Pezzoli C, Galli-Kienle M. Protective role of S-adenosyl-methionine against acetaminophen induced mortality and hepatotoxicity in mice. Biochem Pharmacol 1979;28:3567-3571.

30. Corrales F, Giménez A, Alvarez L, et al. S-adenosylmethionine treatment prevents carbon tetrachloride-induced S-adenosylmethionine synthetase inactivation and attenuates liver injury. Hepatology 1992;16:1022-1027.

31. Simile MM, Banni S, Angioni E, et al. 5-Methylthioadenosine administration prevents lipid peroxidation and fibrogenesis induced in rat liver by carbon-tetrachloride intoxication. J Hepatol 2001;34:386-394.

32. Mato JM, Corrales FJ, Lu SC, Avila MA. S-adenosylmethionine: a control switch that regulates liver function. FASEB J 2002;16:15-26.

33. Lu SC, Mato JM. Role of methionine adenosyltransferase and $\mathrm{S}$-adenosylmethionine in alcohol-associated liver cancer. Alcohol 2005;35:227-234.

34. Osman C, Merkwirth C, Langer T. Prohibitins and the functional compartmentalization of mitochondrial membrane. J Cell Sci 2009;122:3823-3830

35. Ye X, Zhao Y, Zhao LL, et al. Characterization of PHB1 and its role in mitochondrial maturation and yolk platelet degradation during development of artemia embryos. PLoS One 2014;9:e109152.

36. Santamaría E, Avila MA, Latasa MU, et al. Functional proteomics of non alcoholic steatohepatitis: mitochondrial proteins as targets of S-adenosylmethionine. Proc Natl Acad Sci U S A 2003;100:30653070.

37. Jian C, Xu F, Hou T, Sun T, Jinghang L, Cheng H, Wang X. Deficiency of PHB complex impairs respiratory supercomplex formation and activates mitochondrial flashes. J Cell Sci 2017;130:2620-2630.

38. Lu SC, Alvarez L, Huang ZZ, et al. Methionine adenosyltransferase $1 \mathrm{~A}$ knockout mice are predisposed to liver injury and exhibit increased expression of genes involved in proliferation. Proc Natl Acad Sci U S A 2001;98:5560-5565.

39. Pascale RM, Marras V, Simile MM, et al. Chemoprevention of rat liver carcinogenesis by S-adenosyl-L-methionine: a long-term study. Cancer Res 1992;52:4979-4986.

40. Torres L, Avila MA, Carretero MV, et al. Liver-specific methionine adenosyltransferase MAT1A gene expression is associated with a specific pattern of promoter methylation and histone acetylation: implications for MAT1A silencing during transformation. FASEB J 2000;14:95-102.

41. Tomasi ML, Li TW, Li M, Mato JM, Lu SC. Inhibition of human methionine adenosyltransferase $1 \mathrm{~A}$ transcription by coding region methylation. J Cell Physiol 2012;227:1583-1591.

42. Yang H, Huang ZZ, Zeng Z, Chen C, Selby RR, Lu SC. Role of promoter methylation in increased methionine adenosyltransferase 2A expression in human liver cancer. Am J Physiol Gastrointest Liver Physiol 2001;280:G184-190.

43. Frau M, Tomasi ML, Simile MM, et al. Role of transcriptional and posttranscriptional regulation of methionine adenosyltransferases in liver cancer progression. Hepatology 2012;56:165-175.

44. Feo F, De Miglio MR, Simile MM, et al. Hepatocellular carcinoma as a complex polygenic disease. Interpretive analysis of recent developments on genetic predisposition. Biochim Biophys Acta 2006;1765:126-147.

45. Lal A, Mazan-Mamczarz K, Kawai T, Yang X, Martindale JL, Gorospe M. Concurrent versus individual binding of HuR and AUF1 to common labile target mRNAs. EMBO J 2004;23:30923102.

46. Kim MY, Hur J, Jeong S. Emerging roles of RNA and RNA-binding protein network in cancer cells. BMB Rep 2009;42:125-130.

47. Vázquez-Chantada M, Fernández-Ramos D, Embade N, et al. HuR/-methyl-HuR and AUF1 regulate the MAT expressed during liver proliferation, differentiation, and carcinogenesis. Gastroenterology 2010;138:1943-1953.

48. Yang H, Cho ME, Li TW, Peng H, Ko KS, Mato JM, Lu SC. MicroRNAs regulate methionine adenosyltransferase $1 \mathrm{~A}$ expression in hepatocellular carcinoma. J Clin Invest 2013;123:285-298.

49. Yeo EJ, Wagner C. Tissue distribution of glycine $\mathrm{N}$-methyltransferase, a major folate-binding protein of liver. Proc Natl Acad Sci U S A 1994;91:210-214.

50. Heady JE, Kerr SJ. Alteration of glycine N-methyltransferase activity in fetal, adult, and tumor tissues. Cancer Res 1975;35:640-643. 
51. Chen YM, Chen LY, Wong, FH, Lee CM, Chang TJ, Yang-Feng TL. Genomic structure, expression, and chromosomal localization of the human glycine N-methyltransferase gene. Genomics 2000;66:43-47.

52. Avila MA, Berasain C, Torres L, et al. Reduced mRNA abundance of the main enzymes involved in methionine metabolism in human liver cirrhosis and hepatocellular carcinoma. J Hepatol 2000;33:907-914.

53. Chen YM, Shiu JY, Tzeng SJ, et al. Characterization of glycine$\mathrm{N}$-methyltransferase-gene expression in human hepatocellular carcinoma. Int J Cancer 1998;75:787-793.

54. Fernández-Álvarez S, Gutiérrez-de Juan V, Zubiete-Franco I, et al. TRAIL-producing NK cells contribute to liver injury and related fibrogenesis in the context of GNMT deficiency. Lab Invest 2015;95:223-236.

55. Kant R, Yen CH, Lu CK, Lin YC, Li JH, Chen YM. Identification of 1,2,3,4,6-Penta-O-galloyl- $\beta$-d-glucopyranoside as a Glycine $\mathrm{n}$-methyltransferase enhancer by high-throughput screening of natural products inhibits hepatocellular carcinoma. Int J Mol Sci 2016;17.

56. Botezatu A, Bleotu C, Nastase A, et al. Epigenetic silencing of GNMT gene in pancreatic adenocarcinoma. Cancer Genomics Proteomics 2015;12:21-30.

57. Huidobro C, Toraño EG, Fernández AF, et al. A DNA methylation signature associated with the epigenetic repression of glycine $\mathrm{N}$-methyltransferase in human hepatocellular carcinoma. J Mol Med (Berl) 2013;91:939-950.

58. Bhat R, Bresnick E. Glycine N-methyltransferase is an example of functional diversity. Role as a polycyclic aromatic hydrocarbonbinding receptor. J Biol Chem 1997;272:21221-21226.

59. Chen SY, Lin JR, Darbha R, Lin P, Liu TY, Chen YM. Glycine $\mathrm{N}$-methyltransferase tumor susceptibility gene in the benzo(a) pyrene detoxification pathway. Cancer Res 2004;64:3617-3623.

60. Tseng TL, Shih YP, Huang YC, et al. Genotypic and phenotypic characterization of a putative tumor susceptibility gene, GNMT, in liver cancer. Cancer Res 2003;63:647-654.

61. Huang YC, Lee CM, Chen M, et al. Haplotypes, loss of heterozygosity, and expression levels of glycine N-methyltransferase in prostate cancer. Clin Cancer Res 2007;13:1412-1420.

62. Chen M, Huang YL, Huang YC, et al. Genetic polymorphisms of the glycine $\mathrm{N}$-methyltransferase and prostate cancer risk in the health professionals follow-up study. PLoS One 2014;9:e94683.

63. Ottaviani S, Brooke GN, O'Hanlon-Brown C, Waxman J, Ali S, Buluwela L. Characterisation of the androgen regulation of glycine $\mathrm{N}$-methyltransferase in prostate cancer cells. J Mol Endocrinol 2013;51:301-12.

64. Lee CM, Yen CH, Tzeng TY, et al. Androgen response element of the glycine $\mathrm{N}$-methyltransferase gene is located in the coding region of its first exon. Biosci Rep 2013;33.

65. Luka Z, Capdevila A., Mato JM, Wagner C. A Glycine $\mathrm{N}$-methyltransferase knockout mouse model for humans with deficiency of this enzyme. Transgenic Res 2006;15:393-397.

66. Liu SP, Li YS, Chen YJ, et al. Glycine N-methyltransferase-/- mice develop chronic hepatitis and glycogen storage disease in the liver.
Hepatology 2007;46:1413-1425.

67. Liao YJ, Liu SP, Lee CM, Yen CH, et al. Characterization of a glycine $\mathrm{N}$-methyltransferase gene knockout mouse model for hepatocellular carcinoma: Implications of the gender disparity in liver cancer susceptibility. Int J Cancer 2009;124:816-826.

68. Martínez-Chantar ML, Vázquez-Chantada M, Ariz U, et al. Loss of the glycine $\mathrm{N}$-methyltransferase gene leads to steatosis and hepatocellular carcinoma in mice. Hepatology 2008;47:1191-1199.

69. Martínez-López N, García-Rodríguez JL, Varela-Rey M, et al. Hepatoma cells from mice deficient in glycine $\mathrm{N}$-methyltransferase have increased RAS signaling and activation of liver kinase B1. Gastroenterology 2012;143:787-798.

70. Yen $\mathrm{CH}, \mathrm{Lu} \mathrm{YC}, \mathrm{Li} \mathrm{CH}$, et al. Functional characterization of glycine N-methyltransferase and its interactive protein DEPDC6/ DEPTOR in hepatocellular carcinoma. Mol Med 2012;18:286-296.

71. Peterson TR, Laplante M, Thoreen CC, et al. DEPTOR is an mTOR inhibitor frequently overexpressed in multiple myeloma cells and required for their survival. Cell 2009;137:873-886.

72. $\mathrm{Li} \mathrm{CH}$, Yen $\mathrm{CH}$, Chen YF, et al. Characterization of the GNMTHectH9-PREX2 tripartite relationship in the pathogenesis of hepatocellular carcinoma. Int J Cancer 2017;140:2284-2297.

73. Liao YJ, Chen KH, Huang SF, et al. Deficiency of glycine $\mathrm{N}$-methyltransferase results in deterioration of cellular defense to stress in mouse liver. Proteomics Clin Appl 2010;4:394-406.

74. Jencks DA, Mathews RG. Allosteric inhibition of methylenetetrahydrofolate reductase by adenosylmethionine. Effects of adenosylmethionine and NADPH on the equilibrium between active and inactive forms of the enzyme and on the kinetics of approach to equilibrium. J Biol Chem 1987;262:2485-2493.

75. Mudd SH, Cerone R, Schiaffino MC, et al. Glycine $\mathrm{N}$-methyltransferase deficiency: a novel inborn error causing persistent isolated hypermethioninaemia. J Inherit Metab Dis 2001;24:448-464.

76. Augoustides-Savvopoulou P, Luka Z, Karyda S, et al. Glycine $\mathrm{N}$-methyltransferase deficiency: a new patient with a novel mutation. J Inherit Metab Dis 2003;26:745-759.

77. Frau M, Simile MM, Tomasi ML, et al. An expression signature of phenotypic resistance to hepatocellular carcinoma identified by cross-species gene expression analysis. Cell Oncol (Dordr) 2012;35:163-173.

78. Laurent-Puig P, Legoix P, Bluteau O, et al. Genetic alterations associated with hepatocellular carcinomas define distinct pathways of hepatocarcinogenesis. Gastroenterology 2001;120:1763-1773.

79. Lee JS, Chu IS, Mikaelyan A, et al. Application of comparative functional genomics to identify best-fit mouse models to study human cancer. Nat Genet 2004;36:1306-1311.

80. Wang YC, Lin WL, Lin YJ, Tang FY, Chen YM, Chiang EP. A novel role of the tumor suppressor GNMT in cellular defense against DNA damage. Int J Cancer 2014;134:799-810.

81. DebRoy S, Kramarenko II, Ghose S, Oleinik NV, Krupenko SA, Krupenko NI. A novel tumor suppressor function of glycine $\mathrm{N}$-methyltransferase is independent of its catalytic activity but requires nuclear localization. PLoS One 2013;8:e70062. 